\title{
87. Injuries of the Internal Carotid Artery in the Skull
}

\author{
Ryo Takahashi, Yoshio Yamazaki, Rikio Ashikawa \\ and Hiroshi SOEDA \\ E.N.T. Department, Jikei University School of Medicine
}

We would like to describe five cases in which we have experienced bleedings due to injury of the internal carotid artery in the skull for the last several years. Causes of the bleedings were due to the head injury in case 1 , due to a surgury of sinuses in case 2 and due to a surgury of carcinoma of the frontal bone in case 3 , and in other 2 cases it occurred after removing a tumor. The bleedings were seen at the portion where the internal carotid artery is in contact with the ethmoidal sinuses, and their prognoses were good by tampons and bindings of the common carotid artery except cases of the frontal sinus carcinoma and of the hypophyseal tumor.

It is said that many cases of the bleedings from the external carotid artery are encountered in the E.N.T. field. The internal carotid artery, however runs through in contact with the posterior paranasal sinuses, partially the wall of the ethmoidal sinuses and especially in cases which show well-developement of the paranasal sinuses this contact is remarkable.

According to our study in which we have tried to clarify these relations in specimens from 36 cadavers.

The degree of the contact between the sphenoidal sinus or the posterior ethmoid cells and the internal carotid artery;

Severe -19 case
moderate $-8 \prime \prime$
slight $-9 \prime \prime$

The location of the contact between the sphenoidal sinus or the posterior ethmoid cells and the internal carotid artery;
A supralateral part of the sphenoidal sinus
32 cases
A supralateral part of the posterior ethmoid cells
1 case

A supralateral part between the sphenoidal sinus and the posterior ethmoid cells

3 cases

Surgeries around the sphenoidal sinus, therefore, should be prudently done with a sufficient attention to the internal carotid artery, especially in a case which has its well-grown paranasal sinuses.

Such a knowlege as above will be more needed, because surgeries concerning this area are more increased in our modern cities. 